\title{
Velocity Variation Assessment of Red Blood Cell Aggregation with Spectral Domain Doppler Optical Coherence Tomography
}

\author{
XiangQun Xu, ${ }^{1}$ Lingfeng Yu, ${ }^{2}$ and Zhongping Chen ${ }^{2}$ \\ ${ }^{1}$ School of Science, Zhejiang Sci-Tech University, Hangzhou 310018, China; and ${ }^{2}$ Beckman Laser Institute, University \\ of California, Irvine, Irvine, CA 92612, USA \\ (Received 5 January 2010; accepted 5 May 2010; published online 15 May 2010)
}

Associate Editor Jing Bai oversaw the review of this article.

\begin{abstract}
We propose spectral domain Doppler optical coherence tomography (SD-D-OCT) to qualitatively measure red blood cell aggregation. Variance/standard deviation (SD) of the Doppler frequency spectrum in Doppler variance imaging of flowing blood under shearing conditions was developed as a new aggregation index. In in vitro microchannel-flow experiments, porcine blood at various hematocrits with aggregation characteristics induced by dextran 500 or at the presence of plasma fibrinogen was measured by a SD-D-OCT system with a spectrum centered at $1310 \mathrm{~nm}$. The effects of shear rate, hematocrit, aggregation level on the $\mathrm{SD}$ values were investigated. The results demonstrate that Doppler variance imaging using the SD value was capable of differentiating the normal and the aggregated blood with hematocrits of $30-55 \%$ flowing at a shear rate of $40-60 \mathrm{~s}^{-1}$. The SD value was found to be nonsignificant hematocritdependent. It is also a sensitive and repeatable aggregation index for comparison between nonaggregated and aggregated blood samples.
\end{abstract}

Keywords-Doppler optical coherence tomography, Doppler variance image, Red blood cell aggregation, Velocity variation.

\section{INTRODUCTION}

The reversible aggregation of human red blood cells (RBCs) continues to be of interest in the field of hemorheology because RBC aggregation is a major determinant of the in vitro rheological properties of blood and the in vivo flow dynamics and flow resistance of blood. ${ }^{2}$ An abnormally high level of RBC aggregation has been observed in many disease states, such as diabetes, thrombosis, myocardial infarction, vascular diseases, and hematological pathology. ${ }^{9}$ There is now general agreement regarding the correlations

Address correspondence to Xiangqun $\mathrm{Xu}$, School of Science, Zhejiang Sci-Tech University, Hangzhou 310018, China. Electronic mail: xuxiangqun@zstu.edu.cn, jadexu@163.com between elevated levels of fibrinogen or other large plasma proteins and enhanced RBC aggregation and platelet aggregation. ${ }^{22,32} \mathrm{RBC}$ aggregation can also be induced by large macromolecules such as high molecular weight dextrans and other polymers. ${ }^{6}$

RBC aggregation can be assessed using different techniques, including erythrocyte sedimentation rate (ESR), zeta sedimentation rate, low-shear viscometry, image analysis in a flow chamber, ultrasound backscattering, ${ }^{10-12,39}$ and photometry. ${ }^{14,25}$ Recording the intensity of transmitted or back-scattered light under shearing conditions has been widely used to assess different aspects of RBC aggregation. Several commercially available instruments of optical aggregometry in a photometric shearing system have been developed using the principles developed by SchmidSchönbein et al. ${ }^{24}$ In optical aggregometry, the cells should be completely disaggregated, by applying a high shear rate (i.e., 500-600 $\mathrm{s}^{-1}$ ), where they are suddenly exposed to zero-flow conditions or slowed to a shear rate of $3-5 \mathrm{~s}^{-1}$, prior to testing. Light scattering signals, with respect to time, provide information about the cell aggregation characteristics. Even though these methods define meaningful parameters representing $\mathrm{RBC}$ aggregation, these measurements are quite different from the actual flow environment. ${ }^{37}$ The conventional aggregation parameters in terms of an arbitrary unit "M" and "M1" with Myrenne aggregometer and the laser-assisted optical rotational cell analyzer (LORCA) may not directly relate to the in vivo hemorheological characteristics. ${ }^{18}$

Therefore, creating a new principle and method to measure RBC aggregation mimicking actual in vivo flow is of great interest. In our recent study, we found that RBC aggregation of flowing blood within a microchannel caused changes in the variance of optical Doppler frequency spectrum using spectral domain Doppler optical coherence tomography (SD-D-OCT), ${ }^{33}$ 
and this requires further interpretation of the proposed index, i.e., the standard deviation (SD) of Doppler frequency spectrum as an aggregation parameter as well as the measurement principles.

Since the proposed SD of Doppler frequency spectrum as an aggregation parameter may be dependent on shear rate, hematocrit, aggregation level, and geometrical conditions, the present study investigates the effects of these conditions on the SD value of the normal and aggregated blood. In addition, this study evaluates the instrument precision and sensitivity. Our purpose is to validate a SD of the velocity power spectrum as a new aggregation index for qualitative measurement of RBC aggregation.

\section{THEORY}

OCT or Doppler OCT is able to achieve high imaging speed, high velocity sensitivity, and high spatial resolution simultaneously. ${ }^{8,15}$ It has demonstrated considerable potential as a method for static and dynamic imaging of various kinds of tissue and blood. The refractive index mismatching between $\mathrm{RBC}$ cytoplasm and blood plasma is the major source of light scattering in blood. ${ }^{17,26,28}$ The scattering properties of blood are also dependent on RBC volume, shape, and orientation, aggregation and disaggregation capability and hematocrit (HCT). The OCT depth-reflectivity profile has been used to study blood optical clearing that reduces blood scattering by biocompatible agentinduced refractive index matching between RBCs and plasma in order to enhance the capability of light penetration depth, aggregation, sedimentation, and HCT by our and other groups. ${ }^{7,16,17,29,34-36}$ In our previous work, we demonstrated that the slope of the OCT depth-reflectivity profile decreased with the level of RBC aggregation. ${ }^{35,36}$ This is so because RBC aggregation leads to a decrease in scattering of RBCs. ${ }^{21}$ Unfortunately, the relationship between the slope and the aggregation of RBCs could not be directly used because the slope is also HCT-dependent. ${ }^{40}$

We have demonstrated Doppler variance tomography using the variance of the Doppler frequency spectrum to more efficiently map the flows in highly scattering biological tissues by Doppler OCT (D-OCT) ${ }^{31,40}$ In addition to the local velocity information, phase-resolved D-OCT systems give the variance of the Doppler frequency spectrum, ${ }^{31,40}$ which is given by

$$
\sigma^{2}=\frac{2}{T^{2}}\left\{1-\frac{\left|\frac{1}{N} \sum_{j=1}^{N}\left[f_{j T}(z) \cdot f_{(j+1) T}^{*}(z)\right]\right|}{\frac{1}{N} \sum_{j=1}^{N}\left[f_{j T}(z) \cdot f_{j T}^{*}(z)\right]}\right\},
$$

where $N$ is the number of A-lines used for averaging; $T$ is the time interval between two exposures, and $f(z)$ is a complex signal from an inverse Fourier transform of the interference pattern. The corresponding Doppler shift can be estimated by comparing the phasors of $f(z)$ between two complex signals during two exposures at a same location. The phase change is recorded by the product of $f_{j T}(z)$ and $f^{*}{ }_{(j+1) T}(z)$, where * denotes the conjugate operation. The value of $\sigma^{2}$ depends on the flow velocity distribution. Variations of flow velocity broaden the Doppler frequency spectrum and result in a larger $\sigma^{2}$. Thus, the Doppler variance obtained by phase-resolved D-OCT systems can be an indicator of flow variations.

Individual particles within concentrated suspensions exhibit significant random movements when the suspension is subjected to shear flow. These movements are dependent on the local shear rate of the suspension and on particle size. ${ }^{4}$ Previous studies have shown variation in the instantaneous velocity of individual RBCs and the effect of aggregation on the dispersion of RBCs flowing in venules. ${ }^{5,27} \mathrm{RBC}$ aggregation in the flow stream decreases the variability in instantaneous cellular velocity. ${ }^{5}$ This effect is most likely the result of an increased particle size. The magnitude of particle movements is decreased when the particle diameter increases. ${ }^{13,23}$ Therefore, decreased variance/SD in the Doppler frequency spectrum with $\mathrm{RBC}$ aggregation is expected.

\section{MATERIALS AND METHODS}

A schematic of the spectral-domain D-OCT system is shown in Fig. 1. A super-luminescent diode (SLD) with a spectrum centered at $1310 \mathrm{~nm}$, and a full width at half maximum bandwidth of $95 \mathrm{~nm}$ and a total delivered power of $8 \mathrm{~mW}$ was used. Back-reflected light from the reference and sample arms was guided into a spectrometer and dispersed over a $1 \times 1024$ InGaAs detector array at $7.7 \mathrm{kHz}$ (corresponding to

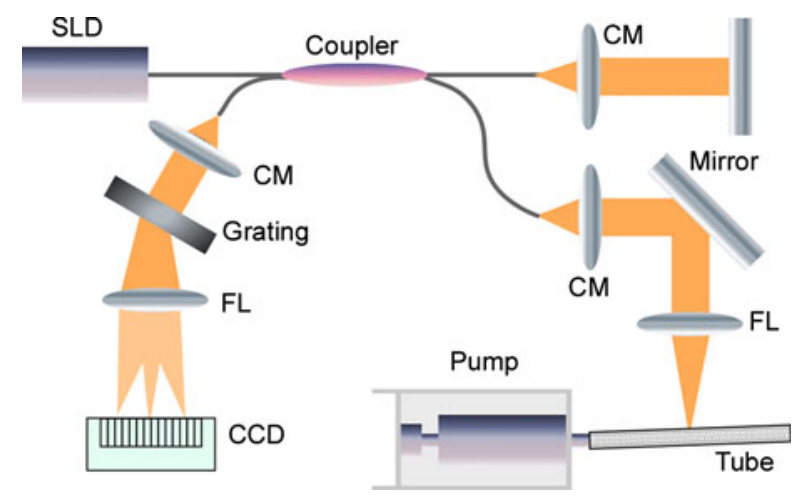

FIGURE 1. Schematic of a fiber-based spectral-domain OCT system used in the experiments ( $F L$, focusing lens; $D G$, diffraction grating; $C M$, collimator; $L S C$, line-scan camera). 
$1 / T)$. The total wavelength range spreading on the detector array was $130 \mathrm{~nm}$, corresponding to a spectral resolution of $0.13 \mathrm{~nm}$ and an imaging depth of $3.6 \mathrm{~mm}$ in air. The resulting axial resolution was about $8 \mu \mathrm{m}$ and the lateral resolution of the system was about $13.8 \mu \mathrm{m} .{ }^{1,36}$

The interference signal was inversely Fourier transformed after reducing the DC component. In the phase-resolved D-OCT, the phase change of the interference fringe between sequential A-line scans was used to calculate the Doppler frequency shift. ${ }^{40}$ The SD of phase shift between two consecutive exposures $(=\sigma T)$ was calculated from the cross correlation using Eq. (1). ${ }^{31}$

Various HCT levels from $30 \%$ to $55 \%$ were obtained by mixing extracted RBCs with porcine plasma using ethylenediaminetetraacetic acid (EDTA) as an anticoagulant (Animal Technologies, Inc., Tyler, TX). Different suspensions of normal RBCs, artificially aggregated with dextran, were prepared. RBCs were resuspended in plasma with dextran 500 (Dx500, average molecular mass $500 \mathrm{kDa}$; Sigma-Aldrich Co., St. Louis, MO, USA). A plasma dextran concentration of $0.5-2 \%$ [in weight $/$ volume $(\mathrm{w} / \mathrm{v})$ ] was used to induce the $\mathrm{RBC}$ aggregation. In another set of experiments, the RBCs were resuspended in PBS for the control samples and in $400 \mathrm{mg} / \mathrm{dL}$ fibrinogen (F4129, SigmaAldrich Co., St. Louis, MO, USA) PBS solution for the aggregating samples at a HCT of $40 \%$. For in vivo situation, fibrinogen or other large plasma proteins enhance RBC aggregation rather than dextran. The experiments are to further demonstrate that the variance can reflect the erythrocyte aggregation mimicking the in vivo situation. All OCT measurements were performed just after RBC suspensions were prepared and pumped through a round glass capillary tube with a 300- $\mu \mathrm{m}$ inner diameter (VitroCom Inc., Mountain Lakes, NJ) at a Doppler angle of $5^{\circ}$. The two ends of the glass tube were connected with two around $50 \mathrm{~cm}-$ long plastic tubes to develop a lamina flow. A programmable syringe pump (Harvard Apparatus, Holliston, MA) was used to make the blood flow with an approximately constant speed at a rate of 1.1, 2.3, and $3.4 \mathrm{~mm} / \mathrm{s}$, corresponding to a pseudo shear rate of 20 , 40 , and $60 \mathrm{~s}^{-1}$. The shear rate simulates the real values within major human retinal venular vessels. OCT "M-scans" (a single A-line repeatedly acquired in time without transverse scanning) were performed. A total of 512 OCT A-lines were acquired in a M-mode scan with the probe beam targeting at the center of the tube. It takes about $66 \mathrm{~ms}$ for the acquisition. Averaging is used to improve the measurement sensitivity. All final results of SD values for both normal and aggregated blood were means of five separate measurements on aliquots of a sample. Each measurement consisted of triplicate data acquired from the same aliquot. A paired $t$ test statistical analysis was applied for the differences in SD between the control group (without dextran) and the treated group (with dextran). For all tests, a level of 0.05 was chosen as a significant level.

Instrument precision for SD parameter was assessed by calculating the coefficient of variation (CV) of 12 measurements on aliquots of normal porcine blood samples with fixed HCT at $40 \%$ at the flow rate of $15 \mu \mathrm{L} / \mathrm{min}$ within the $300 \mu \mathrm{m}$ microchannel; each measurement consisted of triplicate determinations followed by introducing a new aliquot. To determine the sensitivity of the aggregation parameter, two groups of blood samples, i.e., the normal blood group and the blood with $2 \%$ Dx 500 , with fixed HCT at $40 \%$ were measured. The "standardized difference" between these two groups for SD parameter was calculated by dividing the difference between the two groups by the mean of the two standard deviations for that parameter. Note that a higher standardized difference for a parameter indicates that the parameter has a higher power to detect a difference in aggregation.

\section{RESULTS AND DISCUSSION}

\section{Standard Deviation Measurement for RBC Aggregation Characterization}

Figure 2 shows the OCT structural images (Figs. 2a and $2 \mathrm{~b}$ ) and the Doppler variance images that give the variation of the blood velocity distribution for the blood without dextran (Fig. 2c) and the aggregated blood with $0.5 \%$ Dx500 (Fig. 2d) at HCT of $40 \%$ flowing through a 300- $\mu \mathrm{m}$ glass tube at a shear rate of $60 \mathrm{~s}^{-1}$. The different OCT signals between the control blood (Fig. 2a) and the aggregated blood (Fig. 2b) are visualized in the blood with $0.5 \% \mathrm{Dx} 500$. The presence of different velocity variations between the control and the aggregated within the glass tube was observed (Figs. 2(c) and 2d). Note that, although the measured profile of the Doppler velocity fits closely to a parabolic shape, the SD data are not perfectly symmetrical about the $r=0$ axis. The results might be because Doppler velocity is a direct measurement of the phase difference between adjacent A-lines, while the (complex) SD measurement is a function of both the phase and amplitude of adjacent A-lines (according to Eq. 1). The $\mathrm{SD}$ will hold a parabolic distribution if the amplitude is uniform across all cross-sectional A-lines. However, the amplitude is normally not a uniform distribution given the existence of speckle noise and velocity-related motion artifacts (sensitivity reduction) of the flow.

The quantitative values of the SD along the depth calculated according to (1) for the control and the 
(a)

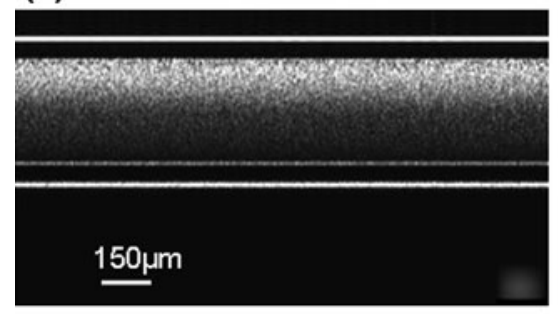

(c)

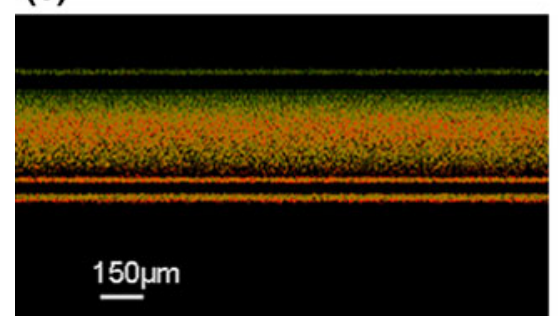

(b)

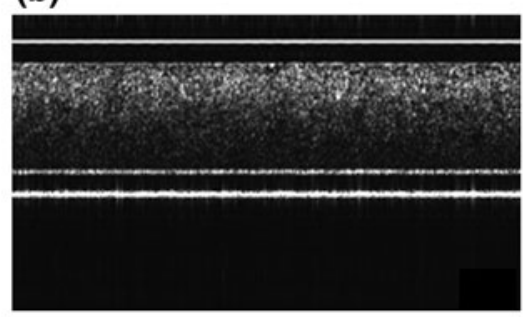

(d)

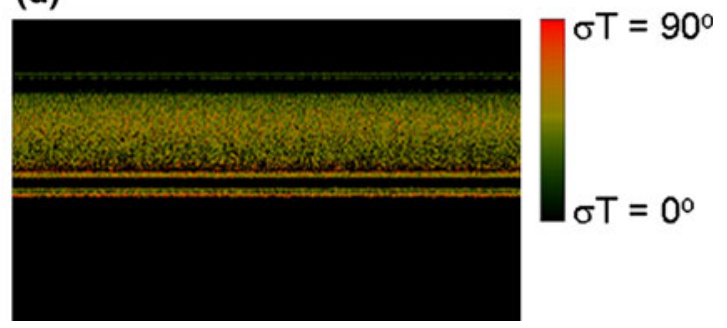

FIGURE 2. Structural OCT images (top) and Doppler variance images (bottom) of the blood at HCT $=40 \%$. (a, c) Normal. (b, d) The blood with $0.5 \%$ dextran 500 .
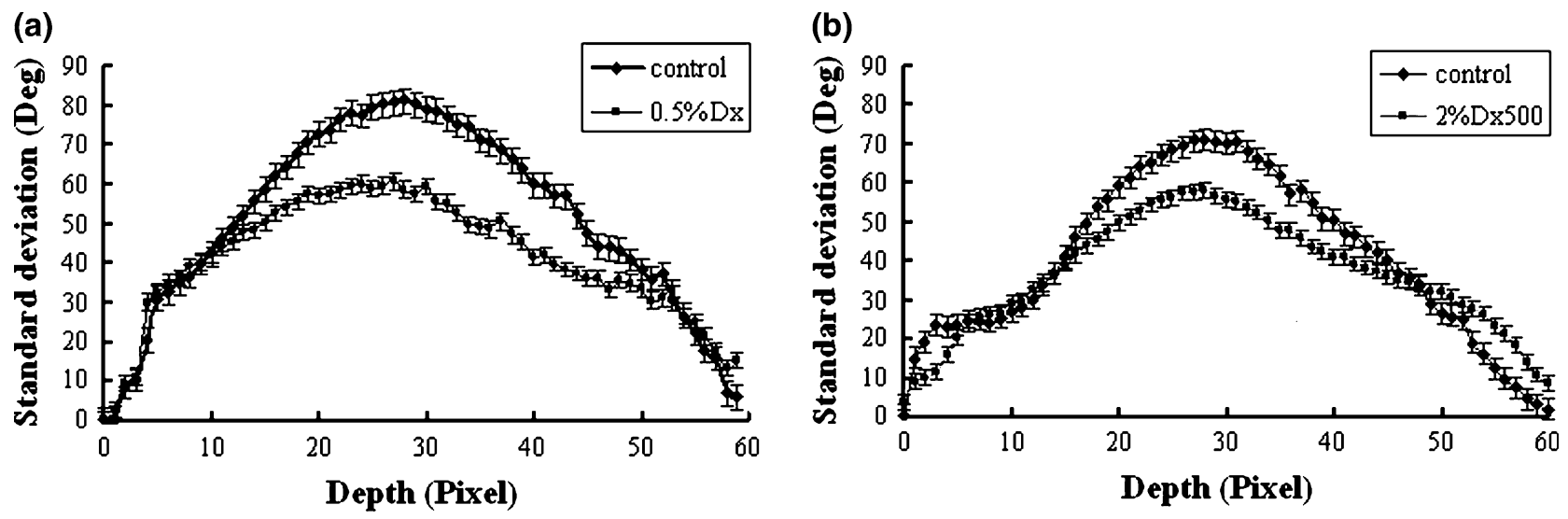

FIGURE 3. Standard deviation vs. depth profiles for normal and dextran-treated blood at HCT: (a) $40 \%$ and (b) $55 \%$.

treated blood were determined and are plotted in Fig. 3 for HCT of $40 \%$ (Fig. 3a) and HCT of $55 \%$ (Fig. 3b) at a shear rate of $60 \mathrm{~s}^{-1}$. For the nonaggregated blood at HCTs of $40 \%$ or $55 \%$, the upper lines of the SD profiles in Figs. $3 a$ and $3 b$ show that the velocity fluctuations vary with radial position in the glass tube. A higher SD is found near the center of the tube where the shear rate is minimal for the flow. The decrease in the SD in the center of the tube could be the result of a decrease in the variability in instantaneous cellular velocity due to changes in the size of scatterers associated with RBC aggregation. When aggregates migrate to the center of the tube, particle size will be greater in the center of the tube than near the tube wall. ${ }^{4}$

The centerline SD values were calculated for each of the RBCs flowing at a shear rate of $60 \mathrm{~s}^{-1}$ and are shown in Fig. 4. The mean values of $74.6 \pm 2.8$ for the control group at a HCT of $40 \%$ is significantly $(p<0.01)$ higher than the values for the aggregated blood induced by dextran from $0.5 \%$ to $2 \%$. The addition of dextran decreases the SD by around $40 \%$, indicating the velocity variation in the aggregated blood is reduced. This finding is consistent with the in vivo work ${ }^{5}$ that the magnitude of the velocity variations in dextran-treated blood is less than that in the nonaggregating blood. It is most likely that RBCs residing in aggregates could be both shielded from interactions with surrounding cells and also hindered in their movements or the magnitude of particle movements is decreased when the particle diameter increases.

It is known that dextran-induced aggregation is higher at higher concentrations up to an optimum 
concentration. ${ }^{20}$ We noted that there is no significant difference the SD values between the samples with dextran at concentrations of $0.5-2 \%$. Because dextran 500 used in our experiment, which has a very high molecular weight, is a strong aggregation inducer producing large aggregates after the concentration over $0.5 \%,{ }^{5,19}$ it seems that the variance cannot distinguish the size difference between the samples with dextran at concentrations of $0.5-2 \%$ after the aggregates exceeding a certain size, i.e., the SD value rapidly drops down to around $45^{\circ}$. Further improvement of the signal-to-noise ratio of the SD-D-OCT system may be helpful to address the issue. Nevertheless, dextran is only used to demonstrate the method and dextran 500 at $0.6 \%$ is widely used to induce $\mathrm{RBC}$ aggregation in

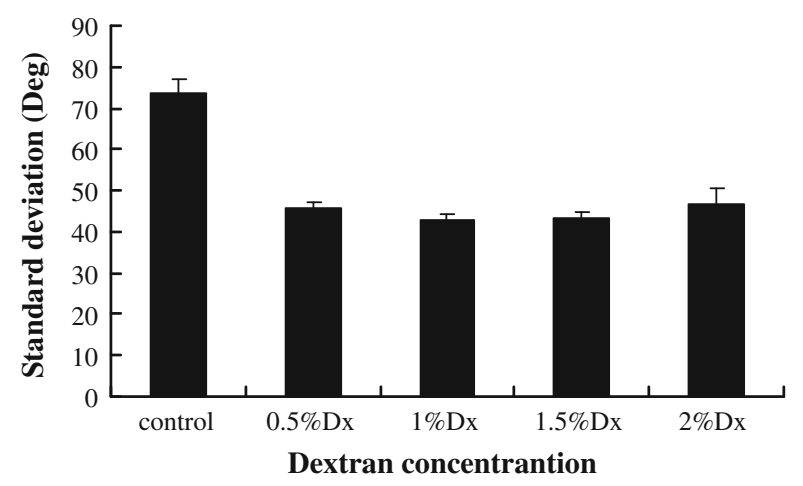

FIGURE 4. Difference in mean centerline SD for the blood (HCT $40 \%$ ) at various concentrations of dextran. Values for dextran-treated blood are significantly $(p<0.01)$ smaller than those for normal blood. the range for healthy humans. ${ }^{5,19}$ Studies on mimicking the in vivo situation with fibrinogen at the measured concentrations and blood samples from patients that the actual aggregation is assessed are under way to develop a quantitative method rather than the current qualitative method.

Figure 5 shows the Doppler variance images that give the variation of the blood velocity distribution for the control (Fig. 5a) and the aggregating blood with fibrinogen (Fig. 5b) at a HCT of $40 \%$ flowing through a $300-\mu \mathrm{m}$ glass tube at a shear rate of $40 \mathrm{~s}^{-1}$. The quantitative values of SD along the depth calculated for the control and the aggregating blood are plotted in Fig. 5c. The presence of different velocity variations between the two groups was evident. The centerline SD for each of the RBCs flowing at shear rates of 40 and $60 \mathrm{~s}^{-1}$ is shown in Fig. 6. Fibrinogen at $400 \mathrm{mg} / \mathrm{dL}$ results in a significant decrease in the SD by $26.7 \%$ $(p<0.01)$ at $40 \mathrm{~s}^{-1}$ and $39.8 \%(p<0.01)$ at $60 \mathrm{~s}^{-1}$, respectively.

\section{Effect of Shear Rate on SD Measurements}

Figure 7 shows the centerline SD of $40 \%$ blood under three shear rates of 20,40 , and $60 \mathrm{~s}^{-1}$ for the normal (nonaggregating) and $2 \%$ Dx500-treated (aggregating) blood. As expected, the SD value increases with increasing shear rate for both groups. When dextran is added to the flowing blood, RBC aggregation causes a significant decrease in the SD values at 40 and $60 \mathrm{~s}^{-1}$. (a)

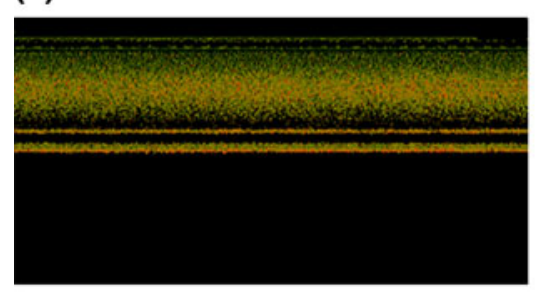

(b)

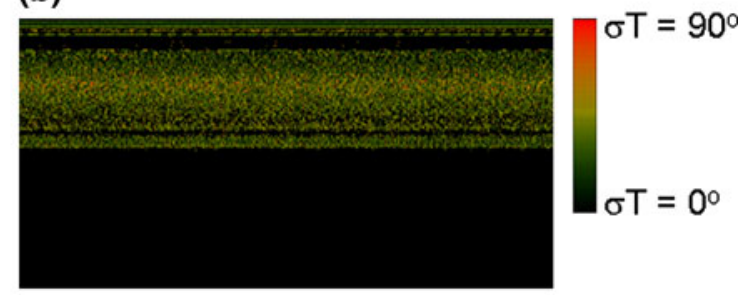

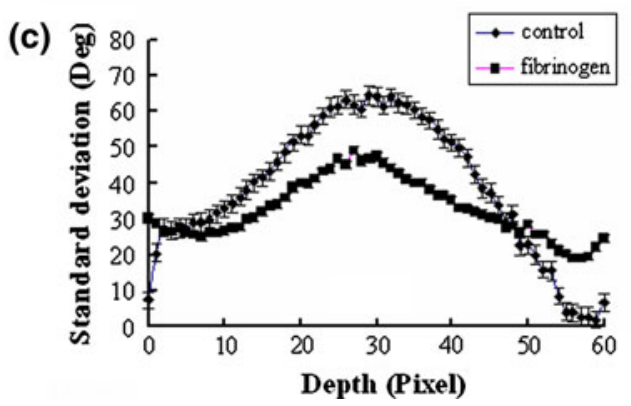

FIGURE 5. Doppler variance images of the normal (a) and the aggregating blood with fibrinogen (b) at HCT of $40 \%$, (c) quantitative values of SD along the depth for the normal and the aggregating blood. 


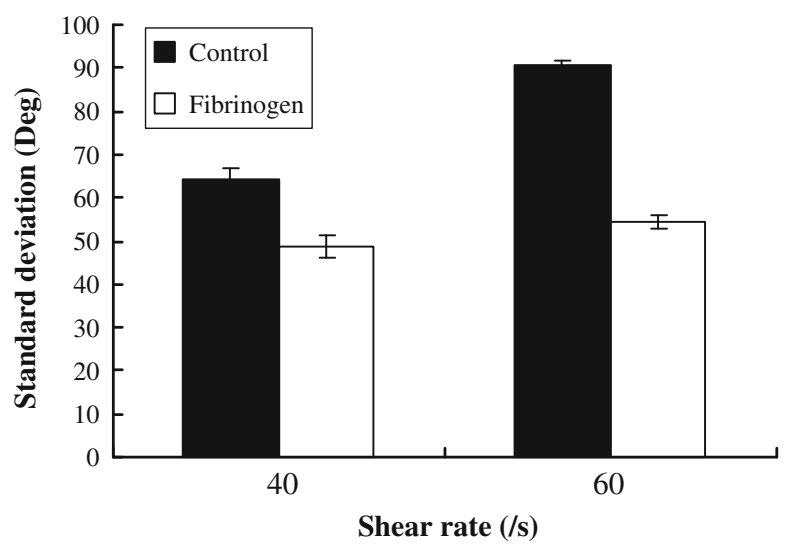

FIGURE 6. Centerline SD values of the normal blood and the blood with fibrinogen at shear rates of 40 and $60 \mathrm{~s}^{-1}$.

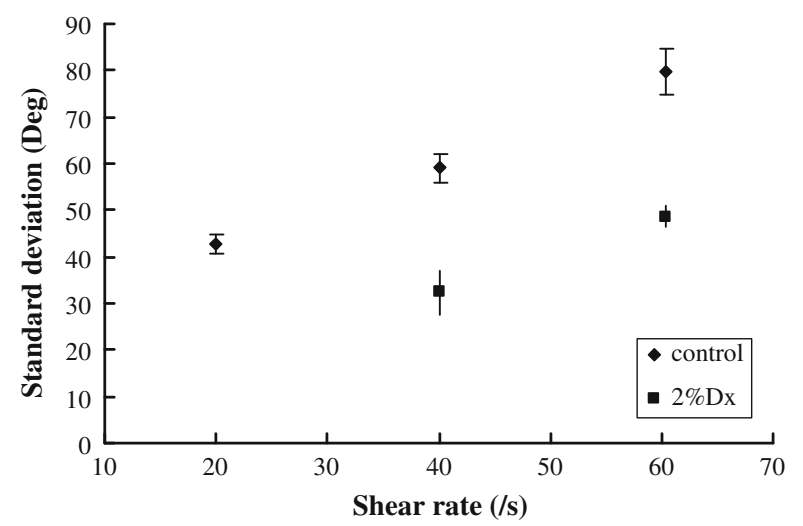

FIGURE 7. Centerline SD values of the blood (HCT 40\%) under three shear rates of 20,40 , and $60 \mathrm{~s}^{-1}$ for the normal blood and $2 \%$ Dx500-treated blood. The value for $20 \mathrm{~s}^{-1}$ with $2 \%$ dextran is not shown in the figure because it drops down to noise level and so meaningless.

\section{Hematocrit Dependence}

Figure 8 shows the centerline SD of control blood with HCTs from $30 \%$ to $55 \%$ at shear rates of 40 and $60 \mathrm{~s}^{-1}$. The SD value tends to decrease with increasing HCT, but that is not significantly affected at the both shear rates. In other words, the SD value is nonsignificant dependence on hematocrits in the range of 30 $55 \%$. The relationship between the SD value and HCT is quite unlike that between scattering and HCT. We and other groups have reported very large changes in attenuation with $\mathrm{HCT}{ }^{16,36}$

\section{Instrument Precision and Sensitivity}

The mean, standard deviation, and the coefficient of variation $(\mathrm{CV})$ for 12 repeated measurements on aliquots of the same control blood sample are $74.6 \%$, $2.8 \%$, and $3.8 \%$, indicating the SD measured by the

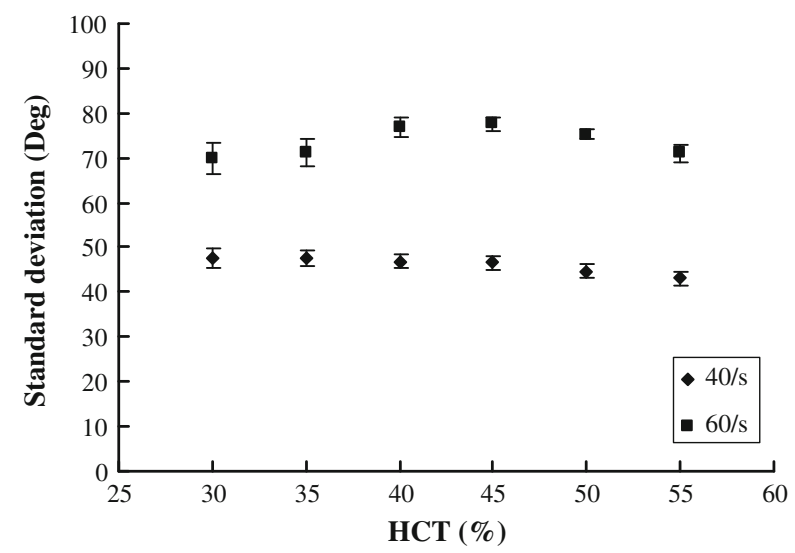

FIGURE 8. Centerline SD values of the control blood at HCTs from $30 \%$ to $55 \%$ at shear rates of 40 and $60 \mathrm{~s}^{-1}$.

TABLE 1. Comparison of instrument precision and sensitivity between Doppler variance method and light transmission method.

\begin{tabular}{lccccc}
\hline Parameter & Mean & $\begin{array}{c}\text { Standard } \\
\text { deviation }\end{array}$ & $\begin{array}{c}\text { CV } \\
(\%)\end{array}$ & $\begin{array}{c}\text { Percentage } \\
\text { difference }\end{array}$ & $\begin{array}{c}\text { Standardized } \\
\text { difference }\end{array}$ \\
\hline $\begin{array}{c}\text { Doppler } \\
\text { variance }\end{array}$ & 74.6 & 2.8 & 3.8 & 60.4 & 8.16 \\
$M_{\text {index }}$ & 24.9 & 1.18 & 4.73 & 75.5 & 2.55 \\
\hline
\end{tabular}

${ }^{\mathrm{a}} \mathrm{M}$ index: integrating the area under the syllectogram curve (light transmission curves) after a sudden stop of shearing.

SD-D-OCT system is highly repeatable. The percentage difference and standardized difference between the two groups ( $n=12$ for each group) of the control and the aggregating blood with $2 \%$ Dx 500 are $60.4 \%$ and 8.16. The standardized difference is much larger than that of commercial available optical aggregometers (Table 1). ${ }^{3}$ The large percentage difference and standardized difference between the two groups implies the $\mathrm{SD}$ value is highly sensitive to aggregation difference.

The data presented in this work point to the possibility of optical Doppler variance determination of the $\mathrm{RBC}$ aggregation. In all our experiments, RBC aggregation induced by dextran or at the presence of fibrinogen caused a practically $30-40 \%$ decrease in the SD of Doppler frequency spectrum. The normal blood has a SD value larger than 70 and the aggregated blood has a SD value smaller than 50 at a pseudo shear rate of $60 \mathrm{~s}^{-1}$. The shear rates between $40-60 \mathrm{~s}^{-1}$ mimicking the venular vessels where $\mathrm{RBC}$ aggregation often exists were found to be optional operating flow velocities for sensitivity, especially higher signal-tonoise ratio at $60 \mathrm{~s}^{-1}$.

One may speculate why the dimension of the round microchannel with an inner diameter of $300 \mu \mathrm{m}$ was used. Typical RBC aggregometers have similar dimensions for their present channel gap. For instance, the LORCA has a $300 \mu \mathrm{m}$ gap in concentric annulus. 
Myrenne aggregometer has an approximately $70 \mu \mathrm{m}$ gap in a cone-plate geometry, which may limit the formation of normal $3 \mathrm{D}$ aggregates.

We have found a new qualitative measure of red blood cell aggregation, directly measured through a $\mathrm{SD}$ of the velocity power spectrum. The new qualitative index function can provide information matching the in vivo microvascular blood flow with shear rates of $40-60 \mathrm{~s}^{-1}$ and is of nonsignificant hematocrit dependence. However, for practical use, a quantitative model relating the $\mathrm{SD}$ to the aggregate size is needed. A wide range of aggregation schemes are possible for RBCs (e.g., diffusion limited aggregation or rouleaux patterns) and understanding their impact on velocity fluctuations is very challenging. Furthermore, as known, the Doppler frequency shift depends on the Doppler angle between the probe and flow directions and would vary during in vivo measurements. However, it was demonstrated that variance imaging is less sensitive to the Doppler angle during in vitro and in vivo measurements in our previous results. ${ }^{30,38}$ Nevertheless, we currently aim to develop an opticalmicrofluidic rheometry with a fixed Doppler angle for in vitro routine medical test. Although the variance of Doppler frequency spectrum is less sensitive to the Doppler angle, the studies on the possible effects on in vivo measurements are under way. Except for the possible effects of the Doppler angle, the phase instability caused by bulk-motion artifacts and optical heterogeneity are also the limitations for in vivo use. A technique to be established using the new index to quantitate $\mathrm{RBC}$ aggregation either for in vitro or in vivo measurements requires further studies.

\section{CONCLUSIONS}

We proposed SD-D-OCT technique to qualitatively measure RBC aggregation with high sensitivity and reproducibility. The SD of Doppler frequency spectrum of RBCs in flowing blood was validated as a new RBC aggregation index, which was independent of blood hematocrit. Clear evidence of correlation between the $\mathrm{SD}$ value of Doppler frequency spectrum and RBC aggregation induced by dextran and fibrinogen which is similar to in vivo blood flow was demonstrated.

\section{ACKNOWLEDGMENTS}

This work was supported by research grants from the National Natural Science Foundation of China (30870675) and Zhejiang Provincial Natural Science Foundation, the National Institutes of Health (EB-00293, CA-91717, and RR-01192), Air Force
Office of Scientific Research (FA9550-0401-0101), and the Beckman Laser Institute Endowment. This work was performed at Dr. Zhongping Chen's OCT lab, Beckman Laser Institute, University of California, Irvine, USA, while Dr. Xiangqun Xu was working there as an academic visitor. The authors thank Dr. Yeh-chan Ahn for discussion.

\section{OPEN ACCESS}

This article is distributed under the terms of the Creative Commons Attribution Noncommercial License which permits any noncommercial use, distribution, and reproduction in any medium, provided the original author(s) and source are credited.

\section{REFERENCES}

${ }^{1}$ Ahn, Y.-C., W. Jung, and Z. Chen. Optical sectioning for microfluidics: secondary flow and mixing in a meandering microchannel. Lab Chip 8:125-133, 2008.

${ }^{2}$ Baskurt, O. K., M. R. Hardeman, H. J. Meiselman, and M. W. Rampling, editors. Handbook of Hemorheology and Hemodynamics, Vol. 69. Amsterdam: IOS Press, pp. H1020-H1032, 2007.

${ }^{3}$ Baskurt, O. K., H. J. Meiselman, and E. Kayar. Measurement of red blood cell aggregation in a "plate-plate" shearing system by analysis of light transmission. Clin. Hemorheol. Microcirc. 19:307-314, 1998.

${ }^{4}$ Bishop, J. J., A. S. Popel, M. Intaglietta, and P. C. Johnson. Rheological effects of red blood cell aggregation in the venous network: a review of recent studies. Biorheoogy 38:263-274, 2001.

${ }^{5}$ Bishop, J. J., A. S. Popel, M. Intaglietta, and P. C. Johnson. Effect of aggregation and shear rate on the dispersion of red blood cells flowing in venules. Am. J. Physiol. Heart Circ. Physiol. 283:H1985-H1996, 2002.

${ }^{6}$ Björn, N., R. Wenby, and H. J. Meiselman. Effects of dextran molecular weight on red blood cell aggregation. Biophys. J. 95:3059-3065, 2008.

${ }^{7}$ Brezinski, M., K. Saunders, C. Jesser, X. Li, and J. G. Fujimoto. Index matching to improve OCT imaging through blood. Circulation 103:1999-2003, 2001.

${ }^{8}$ Chen, Z., T. E. Milner, S. Srinivas, X. Wang, A. Malekafzali, M. J. C. van Germert, and J. S. Nelson. Noninvasive imaging of in vivo blood flow velocity using optical Doppler tomography. Opt. Lett. 22:1119-1121, 1997.

${ }^{9}$ Chien, S., and L. A. Lang. Physicochemical basis and clinical implications of red cell aggregation. Clin. Hemorheol. 7:71-91, 1987.

${ }^{10}$ Cloutier, G., A. Zimmer, F. T. Yu, et al. Increased shear rate resistance and fastest kinetics of erythrocyte aggregation in diabetes measured with ultrasound. Diabetes Care 31(7):1400-1402, 2008.

${ }^{11}$ Fenech, M., D. Garcia, H. J. Meiselman, et al. A particle dynamic model of red blood cell aggregation kinetics. Ann. Biomed. Eng. 37(11):2299-2309, 2009.

${ }^{12}$ Franceschini, E., F. T. H. Yu, and G. Cloutier. Ultrasound characterization of aggregated red blood cells: towards in vivo application. In: Ultrasonic Wave Propagation in 
Nonhomogeneous Media, Springer Proceedings in Physics, Vol. 128. Berlin: Springer, pp. 293-302, 2009.

${ }^{13}$ Goldsmith, H. L., and T. Karino. Microscopic considerations: the motions of individual particles. Ann. NY Acad. Sci. 283:241-255, 1977.

${ }^{14}$ Hardeman, M. R., J. G. G. Dobbe, and C. Ince. Laserassisted optical rotational cell analyser (L.O.R.C.A) as red blood cell aggregometer. Clin. Hemorheol. Microcirc. 25: 1-11, 2001.

${ }^{15}$ Huang, D., E. A. Swanson, C. P. Lin, J. S. Schuman, W. Chang, M. R. Hee, T. Flotte, K. Gregory, C. A. Puliafito, and J. G. Fujimoto. Optical coherence tomography. Science 254:1178-1181, 1991.

${ }^{16}$ Iftimia, N. V., D. X. Hammer, C. E. Bigelow, D. I. Rosen, T. Ustun, A. A. Ferrante, D. Vu, and R. D. Ferguson. Toward noninvasive measurement of blood hematocrit using spectral domain low coherence interferometry and retinal tracking. Opt. Express 14:3377-3388, 2006.

${ }^{17}$ Kirillin, Y., A. V. Priezzhev, V. V. Tuchin, R. K. Wang, and R. Myllylä. Effect of red blood cell aggregation and sedimentation on optical coherence tomography signals from blood samples. J. Phys. D Appl. Phys. 38:2582-2598, 2005.

${ }^{18}$ Lee, B. K., T. Alexy, R. B. Wenby, and H. J. Meiselman. Red blood cell aggregation quantitated via Myrenne aggregometer and yield shear stress. Biorheology 44:29-35, 2007.

${ }^{19}$ Mchedlishvili, G., L. Gobejishvili, and N. Beritasgvili. Effect of intensified red blood cell aggregability on arterial pressure and mesenteric microcirculation. Microvasc. Res. 45:233-242, 1993.

${ }^{20}$ Neu, B., R. Wenby, and H. J. Meiselman. Effects of dextran molecular weight on red blood cell aggregation. Biophys. J. Vol. 95:3059-3065, 2008.

${ }^{21}$ Priezzhev, A. V., O. M. Ryaboshapka, N. N. Firsov, and I. V. Sirko. Aggregation and disaggregation of erythrocytes in whole blood: study by backscattering technique. $J$. Biomed. Opt. 4:76-84, 1999.

${ }^{22}$ Rampling, M. W., H. J. Meiselman, B. Neu, and O. K. Baskurt. Influence of cell-specific factors on red blood cell aggregation. Biorheology 41:91-112, 2004.

${ }^{23}$ Reinke, W., P. Gaehtgens, and P. C. Johnson. Blood viscosity in small tubes: effect of shear rate, aggregation, and sedimentation. Am. J. Physiol. 253:H540-H547, 1987.

${ }^{24}$ Schmid-Schönbein, H., E. Volger, P. Teitel, H. Kiesewetter, U. Dauer, and L. Heilmann. New hemorheological techniques for the routine laboratory. Clin. Hemorheol. 2:93$105,1982$.

${ }^{25}$ Shin, S. J., X. Hou, and J. S. Suh. Measurement of cell aggregation characteristics by analysis of laser-backscattering in a microfluidic rheometry. Korea-Australia Rheol. J. 19:61-66, 2007.

${ }^{26}$ Stover, J. C. Optical Scattering: Measurement and Analysis. Bellingham, WA: SPIE Optical Engineering Press. ISBN 0-8194-1934-6, 1995.
${ }^{27}$ Tangelder, G. J., D. W. Slaaf, A. M. M. Muijtjens, T. Arts, M. G. A. Oude Egbrink, and R. S. Reneman. Velocity profiles of blood platelets and red blood cells flowing in arterioles of the rabbit mesentery. Circ. Res. 59:505-514, 1986.

${ }^{28}$ Tuchin, V. V. Tissue Optics: Light Scattering Methods and Instruments for Medical Diagnosis. Tutorial Texts in Optical Engineering, Vol. TT38. USA: SPIE, 2000.

${ }^{29}$ Tuchin, V. V., X. Xu, and R. K. Wang. Dynamic optical coherence tomography in optical clearing, sedimentation and aggregation study of immersed blood. Appl. Opt. 41:258-271, 2002.

${ }^{30}$ Wang, L. Development of phase-resolved optical Doppler tomography for imaging and quantifying flow dynamics and particle size in microfluidic channels. Ph.D. Dissertation, University of California, Irvine, 2004.

${ }^{31}$ Wang, L., Y. Wang, S. Guo, J. Zhang, M. Bachman, G. P. $\mathrm{Li}$, and Z. Chen. Frequency domain phase-resolved optical Doppler and Doppler variance tomography. Optics Commun. 242:345-350, 2004.

${ }^{32}$ Warwick, S. N., E. Westein, F. J. Tovar-Lopez, E. Tolouei, A. Mitchell, J. Fu, J. Carberry, A. Fouras, and S. P. Jackson. A shear gradient-dependent platelet aggregation mechanism drives thrombus formation. Nat. Med. 15(6): 665-673, 2009.

${ }^{33}$ Xu, X., Y. C. Ahn, and Z. Chen. Feasibility of Doppler variance imaging for red blood cell aggregation characterization. J. Biomed. Opt. 14(6):060507-1-060507-3, 2009.

${ }^{34} \mathrm{Xu}, \mathrm{X}$., R. K. Wang, J. B. Elder, and V. V. Tuchin. Effect of dextran-induced changes in refractive index and aggregation on optical properties of whole blood. Phys. Med. Biol. 48:1205-1221, 2003.

${ }^{35} \mathrm{Xu}, \mathrm{X}$., L. Yu, and Z. Chen. Optical clearing of flowing blood using dextrans with spectral domain optical coherence tomography. J. Biomed. Opt. 13(2):021107-1-0211076, 2008

${ }^{36} \mathrm{Xu}, \mathrm{X}$., L. Yu, and Z. Chen. Effect of erythrocyte aggregation on hematocrit measurement using spectral domain optical coherence tomography. IEEE Biomed. Eng. 55(12): 2753-2758, 2008.

${ }^{37}$ Yalcin, O. M., J. K. Armstrong, H. J. Meiselman, and O. K. Baskurt. Graded alterations of red blood cell aggregation influence in vivo blood flow resistance. Am. J. Physiol. Heart Circ. Physiol. 287:H2644, 2004.

${ }^{38} \mathrm{Yu}$, L., and Z. Chen. Doppler variance imaging for threedimensional retina and choroid angiography. J. Biomed. Optics 15:016029-1-016029-3, 2010.

${ }^{39} \mathrm{Yu}$, F. T., E. Franceschini, B. Chayer, et al. Ultrasonic parametric imaging of erythrocyte aggregation using the structure factor size estimator. Biorheology 46(4):343-363, 2009.

${ }^{40}$ Zhao, Y., Z. Chen, C. Saxer, Q. Shen, S. Xiang, J. F. de Boer, and J. S. Nelson. Doppler standard deviation imaging for clinical monitoring of in vivo human skin blood flow. Opt. Lett. 25(18):1358-1360, 2000. 